\title{
SYSTEMATIC REVIEW AND META-ANALYSIS TO ASSESS THE SAFETY OF BUPROPRION AND VARENICLINE IN PREGNANCY
}

Emily Turner, $\mathrm{BM} \mathrm{BCh}^{1 *}$. Matthew Jones, $\mathrm{PhD}^{1}$. Luis R. Vaz, $\mathrm{PhD}^{1}$. Tim Coleman, $\mathrm{MD}^{1}$.

${ }^{1}$ Division of Primary Care, University Park, University of Nottingham, Nottingham, NG7 2RD, UK

*Corresponding author, emily.turner@nhs.net

WORD COUNT: 3,351 (Abstract 250) 


\section{ABSTRACT}

\section{Introduction}

Smoking in pregnancy is a substantial public health issue, but, apart from nicotine replacement therapy (NRT), pharmacological therapies are not generally used to promote cessation. Bupropion and varenicline are effective cessation methods in non-pregnant smokers and this systematic review investigates their safety in pregnancy.

\section{Methods}

We searched MEDLINE, EMBASE, CINAHL and PsychINFO databases for studies of any design reporting pregnancy outcomes after bupropion or varenicline exposure. We included studies of bupropion used for smoking cessation, depression, or where the indication was unspecified. Depending on study design, quality was assessed using the Newcastle-Ottawa Scale or Cochrane Risk of Bias Tool. Most findings are reported narratively but meta-analyses were used to produce pooled estimates for the proportion of live births with congenital malformations and of the mean birthweight and gestational age at delivery following bupropion exposure.

\section{Results}

18 studies were included: two randomised controlled trials, eleven cohorts, two case-control studies and three case reports. Study quality was variable. Gestational safety outcomes were reported in 14 bupropion and four varenicline studies. Meaningful meta-analysis was only possible for bupropion exposure, for which the pooled estimated proportion of congenital malformations amongst live-born infants was $1.0 \%\left(95 \% \mathrm{Cl}=0.0-3.0 \%, \mathrm{I}^{2}=80.9 \%, 4\right.$ studies $)$ and the mean birthweight and mean gestational age at delivery was $3305.9 \mathrm{~g}\left(95 \% \mathrm{Cl}=3173.2-3438.7 \mathrm{~g}, \mathrm{I}^{2}=77.6 \%, 5\right.$ studies $)$ and 39.2 weeks (95\% $\mathrm{Cl}=38.8-39.6, \mathrm{I}^{2}=69.9 \%, 5$ studies) respectively. 


\section{Conclusions}

There was no strong evidence that either major positive or negative outcomes were associated with gestational use of bupropion or varenicline. PROSPERO registration number CRD42017067064.

\section{IMPLICATIONS}

We believe this to be the first systematic review investigating the safety of bupropion and varenicline in pregnancy. Meta-analysis of outcomes following bupropion exposure in pregnancy suggests that there are no major positive or negative impacts on the rate of congenital abnormalities, birthweight or premature birth. Overall, we found no evidence that either of these treatments might be harmful in pregnancy, and no strong evidence to suggest safety, but available evidence is of poor quality.

\section{INTRODUCTION}

Smoking in pregnancy is associated with increased risks of miscarriage, stillbirth, prematurity, low birth weight, perinatal morbidity and mortality ${ }^{1}$ and is a significant problem in developed countries where rates vary between 8 and $23 \%$ of pregnant women smoking in pregnancy. ${ }^{2-4}$ Children of smoking mothers are twice as likely to become smokers themselves ${ }^{5}$, so smoking in pregnancy and afterwards encourages the persistence of smoking across generations. ${ }^{6}$ Smoking in pregnancy is declining in developed countries but remains highest amongst younger, socially disadvantaged women ${ }^{4}$ and the annual costs of managing the smoking-attributable maternal and infant disease can be substantial. ${ }^{7}$

Studies have shown that pregnancy is the life event which most motivates smokers to attempt cessation, with around half of pregnant smokers attempting to quit. ${ }^{4}$ In addition, although the cost- 
efficacy of smoking cessation interventions in pregnancy is unclear ${ }^{8}$, stopping smoking in pregnancy is likely to save healthcare resources, both with respect to the health of the infant and in the wider context of preventing the perseveration of smoking in the next generation. Thus, promoting smoking cessation during pregnancy will substantially improve the health not only of the infant and mother but of their extended family and, in the longer term, will contribute to reducing the substantial healthcare cost of smoking-related diseases. However, compared to those available for non-pregnant smokers, relatively few effective cessation interventions can be used in pregnancy and nicotine replacement therapy (NRT) is the only drug treatment used to any extent. ${ }^{9}$ The UK National Institute for Healthcare and Excellence (NICE) recommends using NRT, believing it safer that continued smoking in pregnancy. However, in pregnancy NRT has at best only a borderline significant effect on cessation (RR $1.28,95 \% \mathrm{Cl}$ $0.99-1.66)^{10}$ and this lower efficacy, compared to use outside of pregnancy, is probably caused by poor adherence to NRT. ${ }^{11}$

If they were considered sufficiently safe, other effective cessation pharmacotherapies, varenicline and bupropion, could also be tried in pregnancy. Varenicline is well tolerated ${ }^{12}$ and probably more effective than other cessation treatments; ${ }^{13}$ animal research suggests it is not teratogenic. ${ }^{14}$ Similarly, bupropion is an effective smoking cessation aid which approximately doubles non-pregnant smokers chances of stopping. ${ }^{15}$ If varenicline or bupropion were to be proven effective for pregnant smokers, the health benefits which would accrue from stopping smoking would very likely outweigh any minor adverse effects. Consequently, to help assesses whether experimental studies might be ethical, we review evidence for the safety of varenicline and bupropion in pregnancy. 


\section{METHODS}

A study protocol was registered ${ }^{16}$ and the review adhered to Preferred Reporting Items for Systematic Reviews and Meta-Analysis (PRISMA) guidelines. ${ }^{17}$

\section{Inclusion Criteria}

We included studies of any design which reported adverse pregnancy outcomes experienced by mothers, foetuses or infants following use of varenicline or bupropion in pregnancy.

\section{Exclusion criteria}

We excluded studies which presented no empirical data and those in which interventions combined bupropion or varenicline with other cessation pharmacotherapies.

\section{Search Strategy}

We searched the MEDLINE, EMBASE, CINAHL and PsychINFO databases and hand-searched reference lists from reviews and included papers. We also searched for ongoing and unpublished studies at: www.gsk-clinicalstudyregister.com; clincialtrials.gov; www.who.int/trialsearch; www.controlledtrials.com/isrctn; and www.ukctg.nihr.ac.uk. As bupropion and varenicline were licensed and became available relatively recently, we sought studies published from 1990 until $25^{\text {th }}$ May 2017 with no language restrictions. Search terms relating to pregnancy were developed from those used in a Cochrane review ${ }^{10}$ and were combined with qualitative terms relating to smoking, varenicline or bupropion. The protocol also states that we intended to include studies in which women used 'dual' nicotine replacement therapy $(N R T)^{16}$, however only two such studies were identified, and therefore we retrospectively decided that they should not be reviewed separately from other NRT studies (e.g. those investigating 'mono' NRT). 


\section{Data Extraction}

Titles and abstracts were screened by the lead reviewer, retrieving complete manuscripts if necessary to decide on inclusion. All articles were independently assessed by two reviewers to confirm inclusion in the review, with adjudication via a third reviewer when agreement was not met. The following data was extracted by the lead reviewer and checked by a second reviewer, with any discrepancies resolved by a third reviewer: aims and design, numbers of participants, outcomes, data collection, analysis methods and findings. Where studies reported interim analyses, further details were requested from authors.

\section{Quality Assessment}

Quality assessment was performed using Newcastle-Ottawa Scales ${ }^{18}$ for cohort or cross-sectional studies and the Cochrane Risk of Bias Assessment for RCTs. ${ }^{19}$ Initial assessment was made by the lead reviewer and checked by a second reviewer, and discrepancies resolved by a third reviewer. Where a trial had already been quality-assessed for a Cochrane review, we used this published assessment.

\section{Data Synthesis}

A priori, we anticipated that review studies might be so diverse that meaningful data synthesis could be challenging. Hence, we planned making final decisions on whether or not meta-analyses were possible once data extraction was finished, and outcomes of this deliberation are reported alongside review findings. If performed, we anticipated that meta-analyses would be conducted in Stata version $14^{20}$ using a random effects DerSimonian and Laird model to generate pooled means and $95 \%$ confidence intervals with heterogeneity quantified by the $\mathrm{I}^{2}$ statistic. ${ }^{21}$ Rather than not pool studies in the presence of a high $\mathrm{I}^{2}$ value, we planned to present this statistic alongside meta-analysis findings to inform the reader of the extent to which pooled estimates should be treated cautiously. 


\section{RESULTS}

We identified 772 studies (1,053 including duplicates); no ongoing trials were identified from registries and no completed, unpublished studies were identified from pharmaceutical company databases. We identified 30 articles for retrieval in full, with 18 being included in the review (Figure 1); study details and outcomes are shown in Supplementary Material Table 1.

\section{Study Design, Outcome Measures and Quality Assessment}

Included studies comprised two randomised controlled trials (RCTs) ${ }^{22,23}$, eleven cohort studies ${ }^{24-34}$, two case-control studies ${ }^{35,36}$, and three case reports..$^{37-39}$ Maternal or foetal adverse outcomes were reported in fourteen bupropion ${ }^{22-31,36-38}$ and four varenicline studies. ${ }^{32-34,39}$ Although bupropion can be used as an anti-depressant or for smoking cessation, only two RCTs specified that bupropion had been prescribed for smoking cessation..$^{22,23}$ The three observational studies evaluating varenicline exposure did not explicitly state that varenicline was used for smoking cessation, but all discussed its sole indication as a cessation pharmacotherapy. ${ }^{32-34}$

Congenital malformations were reported in eight studies (six following bupropion ${ }^{27-30,35,36}$ and two following varenicline ${ }^{33,34}$ ); reported malformation classification systems are described in Supplementary Material Table 1. Birthweight and gestational age at delivery were reported in five bupropion studies ${ }^{22-}$ 24,26,27. Other outcomes included: foetal loss or stillbirth $25,27,30,32,34$; foetal length or head circumference ${ }^{22,23}$; preterm birth ${ }^{22-24}$; maternal medication adverse effects ${ }^{23,25,39}$; and pre-eclampsia. ${ }^{31}$ An overview of reported outcomes is shown in Table 1.

Study quality was variable (Table 2) with seven of the eleven observational studies assessed as of low methodological quality (score of $<7)^{25,26,29,30,32-34}$; the three case reports were considered to provide only low-quality evidence. ${ }^{37-39}$ 


\section{Potential for Meta-Analyses}

From the distribution of outcomes across studies and study designs (Table 1) it was clear that the few studies investigating varenicline were so different in design and outcome measurement that meaningful meta-analyses was not possible. For bupropion studies, meta-analyses investigating effects on congenital malformation, mean birthweight, and mean gestational age at birth were considered feasible and were undertaken by combining studies or study arms with sufficiently similar designs. For the metaanalysis investigating effects of bupropion exposure on congenital malformations, we included only cohort studies. ${ }^{27-30}$ For birthweight and gestational age at birth meta-analyses, we pooled data from bupropion-exposed arms in cohort studies ${ }^{24,26,27}$ and RCTs $s^{22,23}$ to determine the mean value associated with each outcome.

\section{Bupropion}

\section{Congenital Malformations}

Six studies, four cohort ${ }^{27-30}$ and two case control studies ${ }^{35,36}$, reported congenital malformations. Cohort studies included 3,376 pregnancies (Figure 2) and from these studies the pooled estimate for the percentage of congenital malformations amongst live-born infants exposed to bupropion at any point during gestation was $1.0 \%\left(95 \% \mathrm{Cl}=0.0-3.0 \%, \mathrm{I}^{2}=80.9 \%\right)$ (Figure 3a). As individual studies classified congenital malformations in different ways (Supplementary Material Table 1), we accepted the presence or absence of malformations was as defined within each study and no attempt was made to derive a single classification system applied to all studies. Pregnancies which ended in stillbirth, miscarriage, intra-uterine foetal death or termination were excluded from the analysis, so we defined the proportion of pregnancies with congenital malformations as follows: 
Proportion with any congenital malformation $=\frac{\text { number of live born infants with a malformation }}{\text { total number of live born infants exposed in utero }}$

The two case-control studies had conflicting results. ${ }^{35,36}$ Both used National Birth Defects Prevention Study (NBDPS) criteria $^{40}$ to classify congenital cardiac defects; overall, Alwan found no evidence that maternal bupropion exposure in pregnancy increased infants' risks of developing congenital cardiac defects (adjusted odds ratio $1.4,95 \% \mathrm{Cl}=0.8-2.5) .{ }^{35}$ Alwan did however, report an increased risk of left outflow tract cardiac defects (adjusted odds ratio $2.6,95 \% \mathrm{Cl}=1.2-5.7$ ) which was not found by Louik ${ }^{36}$ (adjusted odds ratio $0.4,95 \% \mathrm{Cl}=0-2.4$ ). Louik investigated the risk of developing eight different cardiac defects following bupropion exposure but did not attempt to estimate the overall risk of any cardiac defect and reported an increased risk of ventricular septal defects (adjusted odds ratio $2.9,95 \% \mathrm{Cl}=1.5$ 5.5) which was not found by Alwan (adjusted odds ratio $1.2,95 \% \mathrm{Cl}=0.5-3.4$ ). Louik found no increased risks for other sub-categories of cardiac defects following bupropion exposure.

\section{Birthweight}

Two RCTs (combined $\mathrm{n}=35$ ) found no significant differences in birthweight between bupropion or placebo groups (Supplementary Material Table 1) but both will likely have been under-powered to detect clinically-significant differences..$^{22,23}$ One controlled cohort study conducted in smokers found significantly higher mean birthweights amongst infants born after exposure to bupropion (3315.9g, SD $553.3, \mathrm{n}=72$ ) compared to those who smoked and used no treatment $(2943.5 \mathrm{~g}, \mathrm{SD} 733.5, \mathrm{n}=900$, $p<0.05) .{ }^{24}$ However, this finding was not replicated in two other bupropion cohort studies. ${ }^{26,27}$ Metaanalysis of the 262 pregnancies in bupropion-exposed arms from cohorts and RCTs gives a pooled estimate for mean birthweight amongst infants exposed to bupropion of $3305.9 \mathrm{~g}(95 \% \mathrm{Cl}=3173.2$ 3438.7g, $\left.\right|^{2}=77.6 \%, n=262$ ) (Figure 3b). 


\section{Gestational Age at Delivery}

Of five studies reporting gestational age at birth, two were RCTs ${ }^{22,23}$ and three cohort studies..$^{24,26,27}$ No significant differences were found between trial groups within the likely under-powered RCTs or between exposure groups in two of the cohort studies. ${ }^{26,27}$ However, one cohort study found that infants born after bupropion exposure had a significantly higher mean gestational age at birth (39.1 weeks, SD 1.3, n=72) compared to non-exposed infants born to smokers (37.5 weeks, SD 3.3, n=900, $p<0.05) .{ }^{24}$ The pooled estimate for mean gestational age at delivery in the five studies which included 260 pregnancies was 39.2 weeks ( $\left.95 \% \mathrm{Cl} 38.8-39.6, \mathrm{I}^{2}=69.9 \%\right)$ (Figure $3 \mathrm{c}$ ).

\section{Foetal Loss}

Three cohort studies reported foetal loss following bupropion exposure $25,27,30$, with only one study including control group data. ${ }^{27}$

The GlaxoSmithKline "Bupropion Pregnancy Registry" cohort reported data from 994 prospectivelyregistered pregnant women (featuring 1005 monitored foetuses) following gestational bupropion exposure. Following first trimester bupropion exposure there were 669 live births, three foetal deaths occurring at or later than 20 weeks gestation, 38 induced abortions, and 96 spontaneous pregnancy losses occurring before 20 weeks. Following second trimester exposure there were 145 live births, one induced abortion and one spontaneous pregnancy loss and after bupropion exposure in the third trimester there were 51 live births and one foetal death. 603 prospectively-registered pregnancies were either lost to follow-up or pending delivery when the register closed, resulting in a loss of outcome data. $^{30}$ 
One study found significantly higher rates of spontaneous abortions $(p=0.009)$ and therapeutic abortions $(p=0.015)$ in a bupropion cohort compared with those exposed to 'non-teratogenic' agents. ${ }^{27} \mathrm{~A}$ small uncontrolled, cohort study of 12 pregnancies, reported five live births, two therapeutic terminations (no explanation of reasons for termination given), one intrauterine death, and four cases that were lost to follow-up following gestational bupropion exposure. ${ }^{25}$

\section{Varenicline}

Four varenicline studies reported relevant adverse outcomes; three cohort studies ${ }^{32-34}$ and one case report study. ${ }^{39}$ No study explicitly stated that such exposures were unintentional, though this was probably the case as there were no smoking cessation studies and varenicline has no therapeutic indications in pregnancy.

Richardson reported outcomes and congenital malformations following exposure to varenicline in pregnancy $(n=89)$ in a study which compared pregnant women exposed to non-teratogenic agents $(n=267)$ with those exposed to either NRT or bupropion (combined group, $n=267$ ). As determined by the EUROCAT classification system for congenital malformations ${ }^{41}$, seven infants $(7.87 \%)$ in the varenicline group were reported to have a congenital malformation; two of which were "major" and five "minor". No significant between-group differences were found in malformation rates. ${ }^{34}$

Another cohort study reported malformation rates in infants both exposed $(4.3 \%, n=254)$ and not exposed to varenicline in utero and also in those exposed to maternal smoking during gestation (4.2\%, $\mathrm{n}=5296)$, and those exposed to neither varenicline nor smoking in utero $(4.2 \%, n=656,139)$. Rates appeared similar but no statistical comparison of groups was undertaken. ${ }^{33}$ 
One uncontrolled cohort of 23 varenicline-exposed pregnancies reported 14 live full-term births (61\%), two live pre-term (<37 weeks gestation) births (9\%), four terminations of pregnancy (17\%), two spontaneous or missed abortions (9\%) and one ectopic pregnancy (4\%). Within this cohort five foetal adverse events were reported, as shown in Supplementary Material Table $1 .^{32}$

One case report described a normal pregnancy, delivery and infant health until six months following gestational varenicline exposure for four weeks from the last menstrual period. ${ }^{39}$

\section{DISCUSSION}

We believe this is the first systematic review investigating the safety of bupropion and varenicline in pregnancy. We found no evidence that either of these treatments might be harmful in pregnancy but available evidence is of poor quality and there is also no strong evidence to suggest safety. Most studies investigated outcomes following bupropion exposure and pooled estimates for birthweight, gestation at birth and congenital abnormality rates do not suggest that any of these outcomes are adversely affected. However, estimates' confidence intervals were relatively wide and more data would be required to improve precision. Far fewer studies investigated outcomes following varenicline exposures and overall there is probably insufficient evidence to make firm conclusions about the safety on any of either therapy in pregnancy.

This study has some limitations. Relatively few studies were eligible for inclusion reflecting a paucity of relevant data and the majority of those in the review were small and observational, with only two RCTs. ${ }^{22,23}$ This restricted assessment of potential causal relationships. Included studies generally had low methodological quality; some are case reports ${ }^{37-39}$ or cohort studies which lack control groups. ${ }^{25,30,32}$ Relatively few studies reported similar outcomes, restricting the potential for meta-analysis and, where 
these were conducted, heterogeneity was high, presumably due to differences between study designs and comparator groups; this heterogeneity means that pooled estimated need to be treated with caution. As there were so few studies investigating bupropion specifically for smoking cessation purposes $^{22,23}$, we combined those which stated explicitly that bupropion had been used for smoking cessation and also those where the purpose of bupropion use was unspecified and this could have been prescribed for either smoking cessation or depression. Consequently, some of the data in our metaanalyses will have come from non-smokers who would be expected to have better birth outcomes than smokers. As the evidence regarding the safety of varenicline during pregnancy is sparser than that for bupropion, with safety data identified in only four studies ${ }^{32-34,39}$, this review predominantly focuses on bupropion. Because of the low quality designs (e.g. uncontrolled ${ }^{32}$ ) and complex comparator groups (e.g. pregnant women exposed to non-teratogenic agents or those using either NRT or bupropion as a single group), no conclusion as to the safety of varenicline can be made. ${ }^{34}$

A strength of this review is its novelty and systematic approach. By including studies with any design it is likely we have identified the majority of available safety evidence. Additionally, the rigorous quality assessment indicates that further investigation of the safety of pharmacotherapy during pregnancy is required. We have aimed to maximise use of available data and believe we have made the best use of this whilst also being sensitive to the limitations inherent in empirical studies' designs.

Our pooled estimate for the proportion of congenital malformations in live-born infants following gestational bupropion exposure (1\%) is similar to those reported in comparable populations. From 20112015 EUROCAT, a European network of population-based registries, reported a congenital abnormalities rate of $2.5 \%$ amongst live births, foetal deaths, stillbirths, and terminations for foetal abnormalities. ${ }^{42}$ In addition, the MACDP (Metropolitan Atlanta Congenital Defects Program) determined the rate in five central counties of Atlanta between 1968 and 2003, to be $2.67 \%{ }^{43}$ Included papers reported only abnormalities within live-born infants; however population-based registries generally include 
pregnancies in which the fetus dies before term. As abnormalities are less likely to be present in liveborn infants, the $1 \%$ review-derived rate may underestimate prevalence in all pregnancies and the interpretation of our data is not completely straightforward. Despite this, it is reassuring that the upper $95 \% \mathrm{Cl}$ for the estimate (3\%) is close to population estimates; further studies would increase the precision of this estimate and possibly provide further reassurance that congenital abnormality rates are not higher after bupropion exposure.

Although 95\% confidence intervals are consistent with wide range of values, the meta-analysis derived point estimate for mean birthweight following bupropion exposure 3305.9g (95\% Cl: 3173.2-3438.7g) was similar to the population average of the countries in which the studies reporting this outcome were conducted. Studies included in the birthweight meta-analysis were predominantly North American and only one was UK-based. ${ }^{27}$ Population-based data show that the average birthweight for those born between 37 and 41 weeks of gestation in the U.S.A. in 2005 was 3389g (SD 466) ${ }^{44}$, and in 2009 the mean birthweight of Canadian babies was 3364g. ${ }^{45}$ Calculating the effects of bupropion on birthweight is also complicated by the known reduction in birthweight associated with maternal smoking; for example, one large study of 3,338 mothers reported an adjusted birthweight deficit within babies born to active smokers averaging 226 grams. ${ }^{46}$ Four of the studies contributing to the pooled estimate for birthweight following bupropion exposure included only pregnant smokers $\mathrm{s}^{22-24,26}$ and the remaining study controlled for the effects of smoking by matching study groups by smoking status. ${ }^{27}$ None of the review studies reported a mean birthweight within the bupropion-exposed groups that was significantly less than their control groups $22-24,26,27$; in 4 studies, birthweights were higher in the bupropion cohorts $22,23,26,27$, and in one study this finding was statistically significant. ${ }^{24}$ The latter study reported increasingly heavier birthweights between pregnant smokers who used no cessation pharmacotherapy, who used a nicotine patch, and who used bupropion, with rates of smoking cessation during pregnancy of $0 \%, 79 \%$ and $81 \%$, respectively. The high rates of smoking cessation in the bupropion-exposed cohort in this study may 
have been the driving factor behind the higher birthweight within the group, rather than being associated with bupropion pharmacotherapy itself, but this nonetheless is a beneficial outcome.

We calculated the pooled mean gestational age at delivery following bupropion exposure as 39.2 weeks (95\% $\mathrm{Cl} 38.8-39.6)$, as shown in Figure 3. This is comparable to the normal 40 week gestation and clinically insignificant. When assessing the studies which compared bupropion-exposed infants to pregnant smokers not using bupropion, there was also no evidence of a significant negative effect. One study found the mean gestational age at birth for infants born to pregnant smokers using bupropion was significantly later than that of pregnant smokers using nicotine patch or no cessation pharmacotherapy, which may be in some part associated with higher smoking cessation rates within the bupropion exposed group. ${ }^{24}$ The remainder of the studies either found no significant differences in mean gestational age at delivery ${ }^{22,26}$ or reported similar findings between exposed and non-exposed groups with no determination of significance levels. ${ }^{23,27}$

Whilst this review demonstrates the paucity of safety evidence, the authors are aware of several ongoing studies which will provide further insight. These include the Australian "Smoking MUMS Study", a population-based investigation to further assess the safety of these agents in pregnancy ${ }^{47}$, two investigating bupropion ${ }^{48,49}$, and one of varenicline..$^{50}$

\section{CONCLUSION}

This review finds no conclusive evidence for the safety of gestational use of bupropion or varenicline. Pooling the limited available evidence suggests that bupropion has no major positive or negative impacts on the rates of congenital abnormalities, birthweight or premature birth. 


\section{Contributors}

$E T, M J, L C$ and TC were involved in the development of the research question. ET performed the electronic searches and initial screening by title and abstract. Articles were reviewed independently by ET and one of MJ, LC or TC, and agreement was sought on whether or not these met inclusion criteria. If required, consensus was achieved by consulting a third author. Data extraction was completed by ET and checked by MJ, LC or TC, with discrepancies resolved by consensus or by involving a third researcher, where necessary. ET was responsible for conducting the qualitative review. ET, MJ, LZ and TC all contributed to the drafting of the final manuscript.

\section{Funding}

No funding was sought to undertake this review.

\section{Declaration of Interests}

The authors declare no conflicts of interest.

\section{Acknowledgments}

The authors would like to thank April McCambridge for her assistance with this review.

Professor Coleman is a National Institute for Health Research (NIHR) Senior Investigator. The views expressed in this article are those of the author(s) and not necessarily those of the NHS, the NIHR, or the Department of Health and Social Care 


\section{REFERENCES}

1. Clarke S, Woodcock A, Bewley B. Smoking and the Young - Summary of a Report of a Working Party of the Royal College of Physicians. J R Coll Physicians of Lond. 1992;26(4):352-356.

2. Curtin SC, Mathews TJ. Smoking Prevalence and Cessation Before and During Pregnancy: Data From the Birth Certificate, 2014. CDC NCHS NVSS. 2016;65(1).

3. Cui Y, Shooshtari S, Forget EL, et al. Smoking during Pregnancy: Findings from the 2009-2010 Canadian Community Health Survey. PLoS ONE. 2014;9(1):e84640.

4. McAndrew F, Thompson J, Fellows L, et al. Infant Feeding Survey 2010. The NHS HSE Info Centre; 2012.

5. Leonardi-Bee J, Jere ML, Britton J. Exposure to parental and sibling smoking and the risk of smoking uptake in childhood and adolescence: a systematic review and meta-analysis. Thorax. 2011;66(10):847.

6. Roberts KH, Munafò MR, Rodriguez D, et al. Longitudinal Analysis of the Effect of Prenatal Nicotine Exposure on Subsequent Smoking Behavior of Offspring. Nicotine Tob Res. 2005;7(5):801-808.

7. Godfrey C. Estimating the Costs to the NHS of Smoking in Pregnancy for Pregnant Women and Infants. PHRC: University of York. 2010.

8. Ruger JP, Weinstein MC, Hammond SK, et al. Cost-effectiveness of motivational interviewing for smoking cessation and relapse prevention among low-income pregnant women: a randomized controlled trial. Value Health. 2008;11(2):191-198.

9. Dhalwani NN, Szatkowski L, Coleman T, et al. Prescribing of nicotine replacement therapy in and around pregnancy: a population-based study using primary care data. Br J Gen Pract. 2014;64(626):e554-560.

10. Coleman T, Chamberlain C, Davey MA, et al. Pharmacological interventions for promoting smoking cessation during pregnancy. Cochrane Database Syst Rev. 2015(12):CD010078.

11. Vaz LR, Aveyard P, Cooper S, et al. The Association Between Treatment Adherence to Nicotine Patches and Smoking Cessation in Pregnancy: A Secondary Analysis of a Randomized Controlled Trial. Nicotine Tob Res. 2016;18(10):1952-1959.

12. Kotz D, Viechtbauer W, Simpson C, et al. Cardiovascular and neuropsychiatric risks of varenicline: a retrospective cohort study. Lancet Respir Med. 2015;3(10):761-768.

13. Cahill K, Stead LF, Lancaster T. Nicotine receptor partial agonists for smoking cessation. Cochrane Database Syst Rev. 2012(4).

14. Agency EM. Champix : EPAR - Scientific Discussion. Pub European Medicines Agency. 2006.

15. Wu P, Wilson K, Dimoulas P, et al. Effectiveness of smoking cessation therapies: a systematic review and meta-analysis. BMC Public Health. 2006;6:300-300.

16. Turner E, Jones M, Vaz L, et al. Emily Turner, Matthew Jones, Luis Vaz, Tim Coleman. A systematic review to assess the safety of drug treatments which are used rarely for smoking cessation in pregnancy: dual nicotine replacement therapy, varenicline and bupropion. PROSPERO 2017:CRD42017067064. PROSPERO. 2017.

17. Liberati A, Altman DG, Tetzlaff J, et al. The PRISMA Statement for Reporting Systematic Reviews and Meta-Analyses of Studies That Evaluate Health Care Interventions: Explanation and Elaboration. PLOS Medicine. 2009;6(7):e1000100.

18. Wells G SB, O'Connell D, Peterson J, et al. The Newcastle-Ottawa Scale (NOS) for assessing the quality of nonrandomised studies in meta-analyses. Available at: http://www.ohri.ca/programs/clinical_epidemiology/oxford.asp. Accessed 07.05.2017

19. Higgins JPT, Altman DG, Gøtzsche PC, et al. The Cochrane Collaboration's tool for assessing risk of bias in randomised trials. BMJ. 2011;343. 
20. StataCorp Stata Statistical Software. Release 14.0 edn. College Station TSL, 2015.

21. DerSimonian R, Laird N. Meta-analysis in clinical trials. Control Clin Trials. 1986;7(3):177-188.

22. Nanovskaya TN, Oncken C, Fokina VM, et al. Bupropion sustained release for pregnant smokers: a randomized, placebo-controlled trial. Am J Obstet Gynecol. 2017;216(4):420.e421-420.e429.

23. Stotts AL, Northrup TF, Cinciripini PM, et al. Randomized, controlled pilot trial of bupropion for pregnant smokers: challenges and future directions. Am J Perinatol. 2015;32(4):351-356.

24. Berard A, Zhao JP, Sheehy O. Success of smoking cessation interventions during pregnancy. Am J Obstet Gynecol. 2016;215(5):611.e611-611.e618.

25. Boshier A, Wilton LV, Shakir SA. Evaluation of the safety of bupropion (Zyban) for smoking cessation from experience gained in general practice use in England in 2000. Eur J Clin Pharmac. 2003;59(10):767-773.

26. Chan B, Einarson A, Koren G. Effectiveness of bupropion for smoking cessation during pregnancy. J Addict Dis. 2005;24(2):19-23.

27. Chun-Fai-Chan B, Koren G, Fayez I, et al. Pregnancy outcome of women exposed to bupropion during pregnancy: a prospective comparative study. Am J Obstet Gynecol. 2005;192(3):932-936.

28. Cole JA, Modell JG, Haight BR, Cosmatos IS, Stoler JM, Walker AM. Bupropion in pregnancy and the prevalence of congenital malformations. Pharmacoepidemiol Drug Saf. 2007;16(5):474-484.

29. Einarson A, Choi J, Einarson TR, Koren G. Incidence of major malformations in infants following antidepressant exposure in pregnancy: results of a large prospective cohort study. Can $J$ Psychiatry. 2009;54(4):242-246.

30. GlaxoSmithKline. The Bupropion Pregnancy Registry: Final Report. 2008. Available at: http://pregnancyregistry.gsk.com/documents/bup_report_final_2008.pdf. Accessed 27.05.17.

31. Palmsten K, Huybrechts KF, Michels KB, et al. Antidepressant use and risk for preeclampsia. Epidemiology. 2013;24(5):682-691.

32. Harrison-Woolrych M, Paterson H, Tan M. Exposure to the smoking cessation medicine varenicline during pregnancy: a prospective nationwide cohort study. Pharmacoepidemiol Drug Saf. 2013;22(10):1086-1092.

33. Olsen M, Petronis KR, Froslev T, et al. Maternal use of varenicline and risk of congenital malformations. Pharmacoepidemiol Drug Saf. 2015;24:244.

34. Richardson JL, Stephens S, Yates LM, et al. Pregnancy outcomes after maternal varenicline use; analysis of surveillance data collected by the European Network of Teratology Information Services. Reprod Toxicol. 2017;67:26-34.

35. Alwan S, Reefhuis J, Botto LD, et al. Maternal use of bupropion and risk for congenital heart defects. Am J Obstet Gynecol. 2010;203(1):52.e51-56.

36. Louik C, Kerr S, Mitchell AA. First-trimester exposure to bupropion and risk of cardiac malformations. Pharmacoepidemiol Drug Saf. 2014;23(10):1066-1075.

37. Gisslen T, Nathan B, Thompson T, Rao R. Hyperinsulinism associated with gestational exposure to bupropion in a newborn infant. J Ped Endocrinol Metab. 2011;24(9-10):819-822.

38. Leventhal K, Byatt N, Lundquist R. Fetal cardiac arrhythmia during bupropion use. Acta Obstet Gynecol Scand. 2010;89(7):980-981.

39. Kaplan YC, Olgac Dündar N, Kasap B, et al. Pregnancy Outcome after Varenicline Exposure in the First Trimester. Case Rep Obstet Gynecol. 2014;2014:263981.

40. Reefhuis J, Gilboa SM, Anderka M, et al. The National Birth Defects Prevention Study: a review of the methods. Birth Defects Res A Clin Mol Teratol. 2015;103(8):656-669.

41. EESoC. Guidelines for Registration. Available at: http://www.eurocatnetwork.eu/aboutus/datacollection/guidelinesforregistration/guide1_4. Accessed 06.07.17.

42. EESoC. Prevalence Tables. Available at: http://www.eurocatnetwork.eu/accessprevalencedata/prevalencetables. Accessed 06.07.17. 
43. Program MACD. Metropolitan Atlanta Congenital Defects Program: Executive summary. Birth Defects Res A Clin Mol Teratol. 2007;79(2):66-93.

44. Donahue SMA, Kleinman KP, Gillman MW, et al. Trends in Birth Weight and Gestational Length Among Singleton Term Births in the United States: 1990-2005. Obstet Gynecol. 2010;115(2 Pt 1):357-364.

45. Statistics Canada CVS. Birth Database (CANSIM table 102-4510). Available at http://www5.statcan.gc.ca/cansim/a26?lang=eng\&id=1024510. Accessed 06.07.17.

46. Ellard GA, Johnstone FD, Prescott RJ, et al. Smoking during pregnancy: the dose dependence of birthweight deficits. BJOG: Int J of Obstet Gynaecol. 1996;103(8):806-813.

47. Havard A, Jorm LR, Preen D, et al. The Smoking MUMS (Maternal Use of Medications and Safety) study: Protocol for a population-based cohort study using linked administrative data. BMJ Open. 2013;3 (9)(e003692).

48. Kranzler H. Placebo-controlled Trial of Bupropion for Smoking Cessation in Pregnant Women (BIBS). NLM identifier: NCT02188459. Available from:

https://clinicaltrials.gov/ct2/show/NCT02188459. Accessed 06.07.17.

49. Miller H. Bupropion for Smoking Cessation in Pregnancy. NLM identifier: NCT01875172. Available from: https://clinicaltrials.gov/ct2/show/NCT01875172. Accessed 06.07.17.

50. Pfizer. Varenicline Pregnancy Cohort Study. NLM identifier: NCT01875172.Available from: https://clinicaltrials.gov/ct2/show/NCT01290445. Accessed 06.07.17. 
Figure 1. PRISMA flowchart of included studies

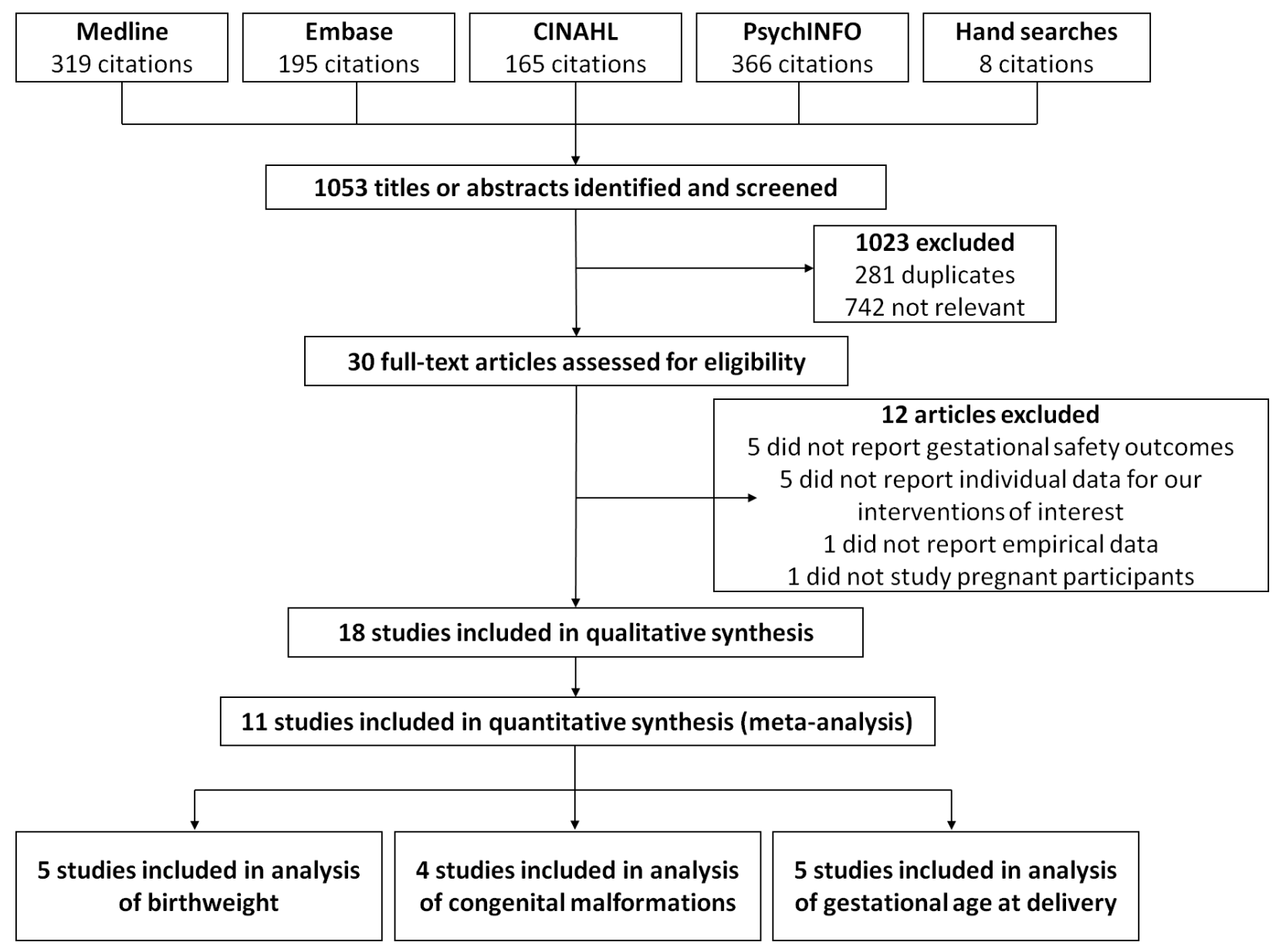

Figure 2. Overview of pregnancies included in meta-analysis of congenital malformations following bupropion exposure

3657 exposed pregnancies identified

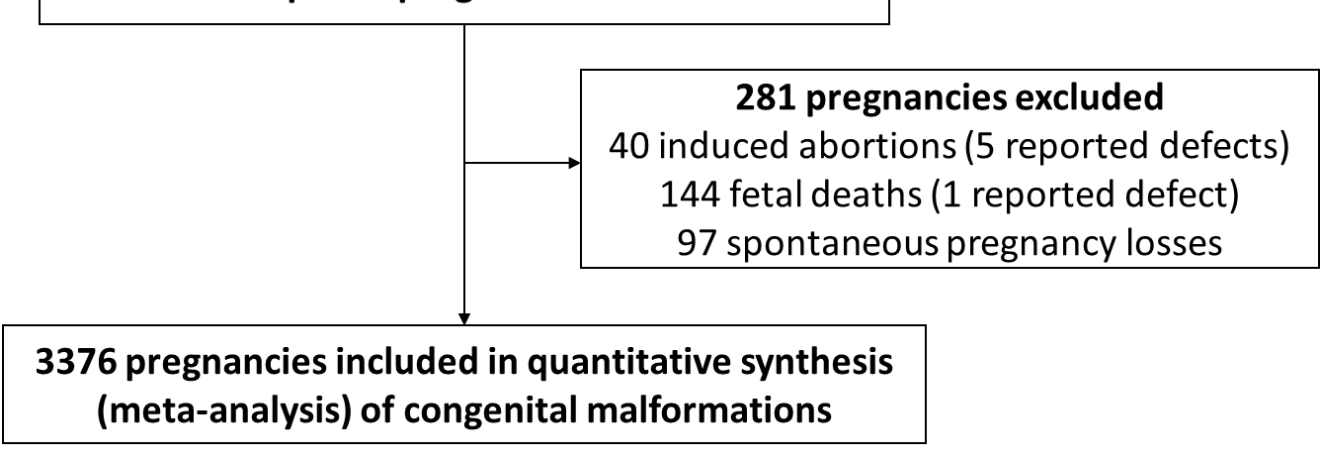


Figure 3a: Proportion of congenital malformations following bupropion exposure

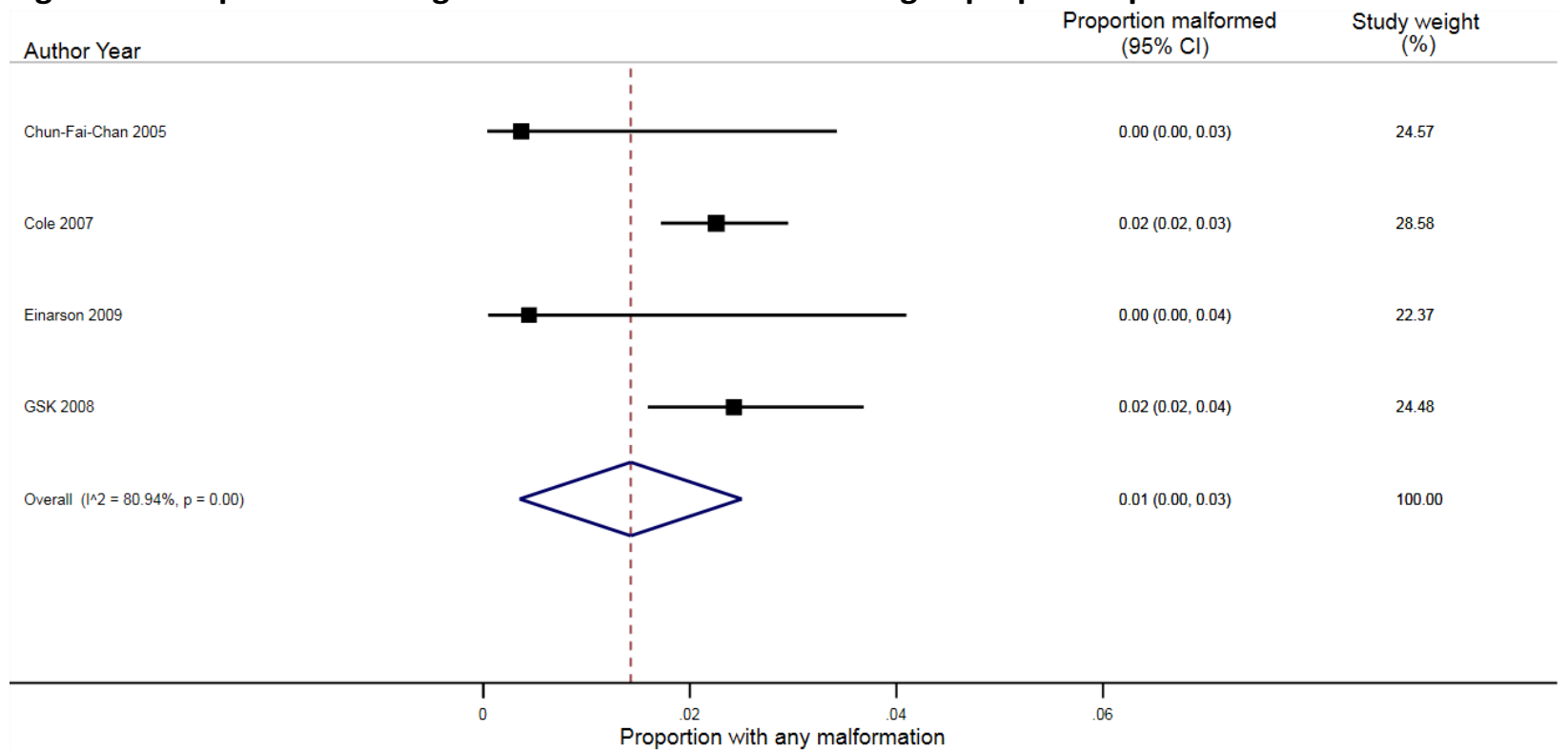

Figure 3b: Mean birthweight following bupropion exposure

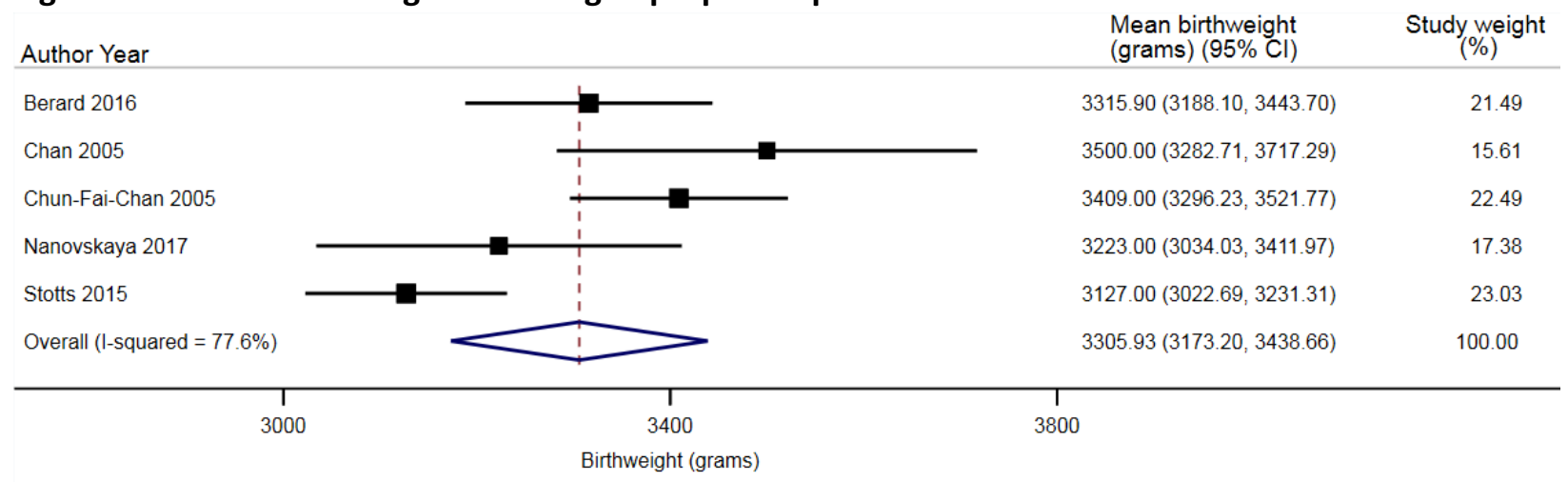

NOTE: Weights are from random-effects model

Figure 3c: Mean gestational age at delivery following bupropion exposure

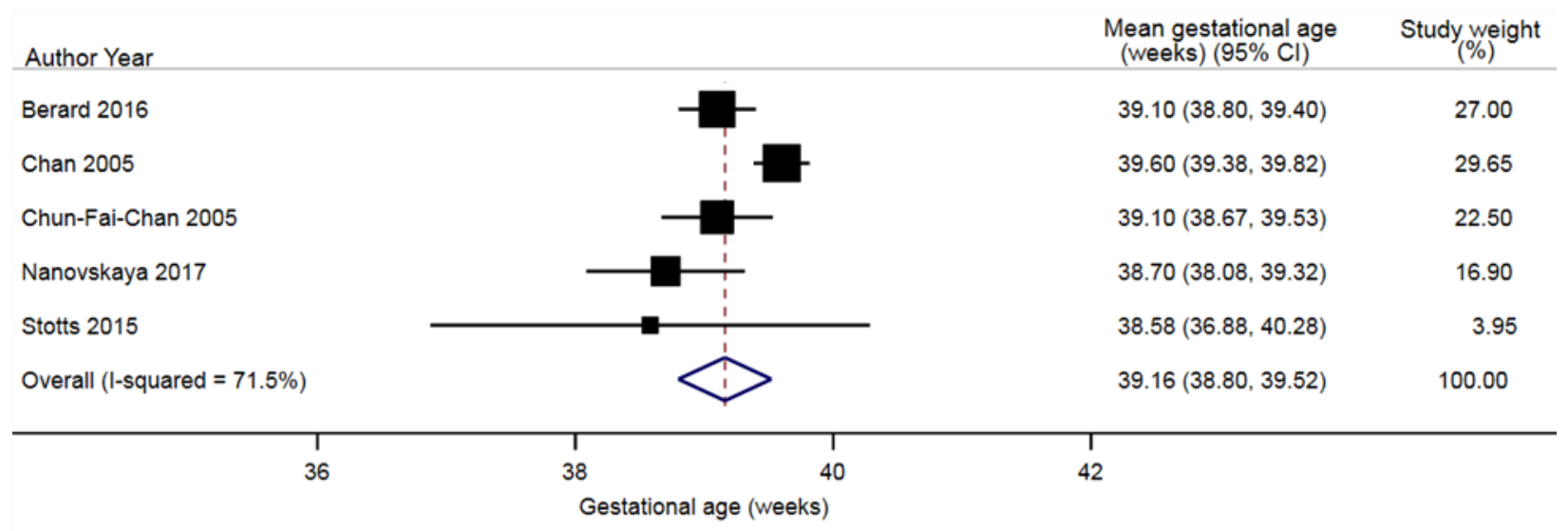

NOTE: Weights are from random-effects model 


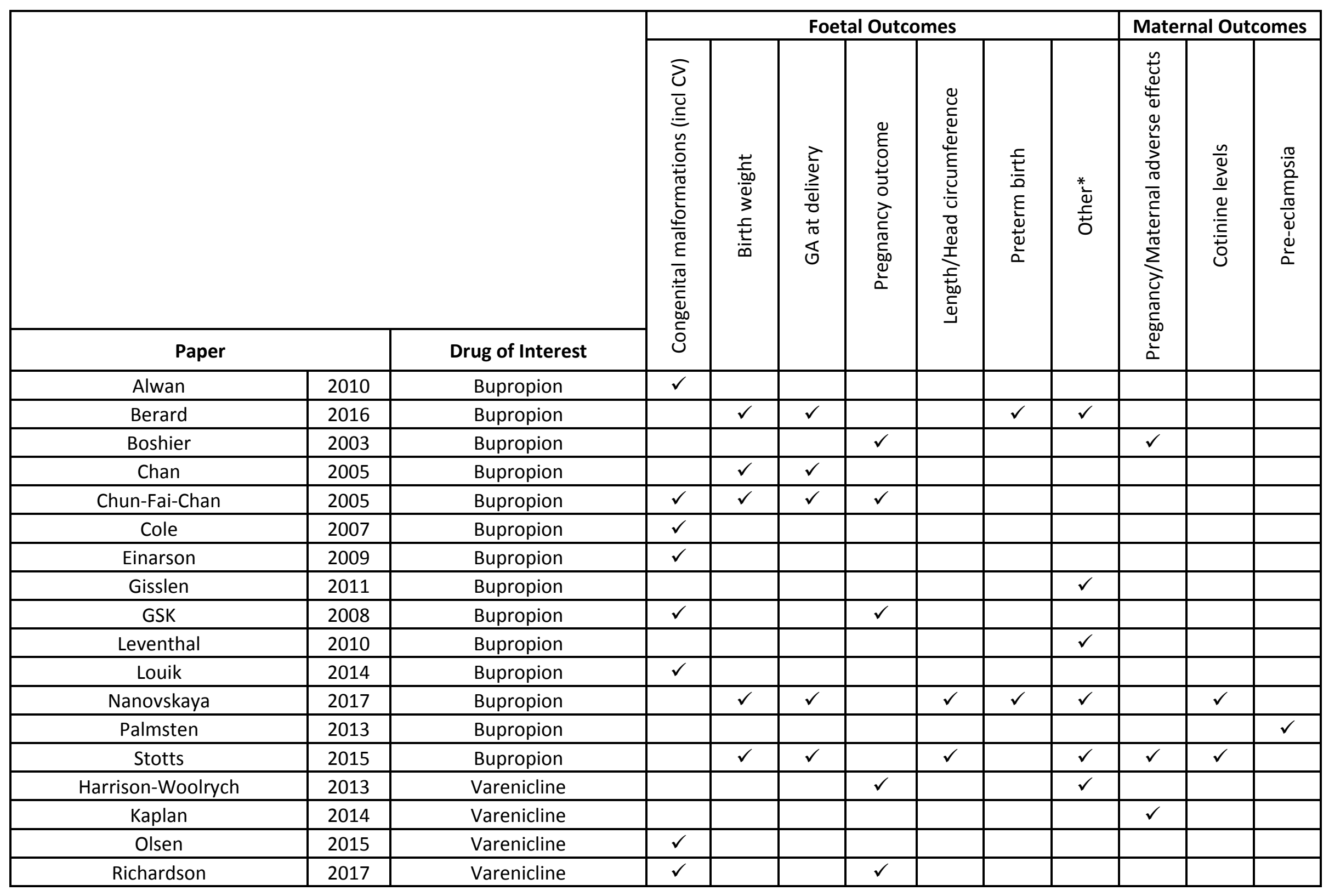


Table 2. Quality Assessment of Included Studies

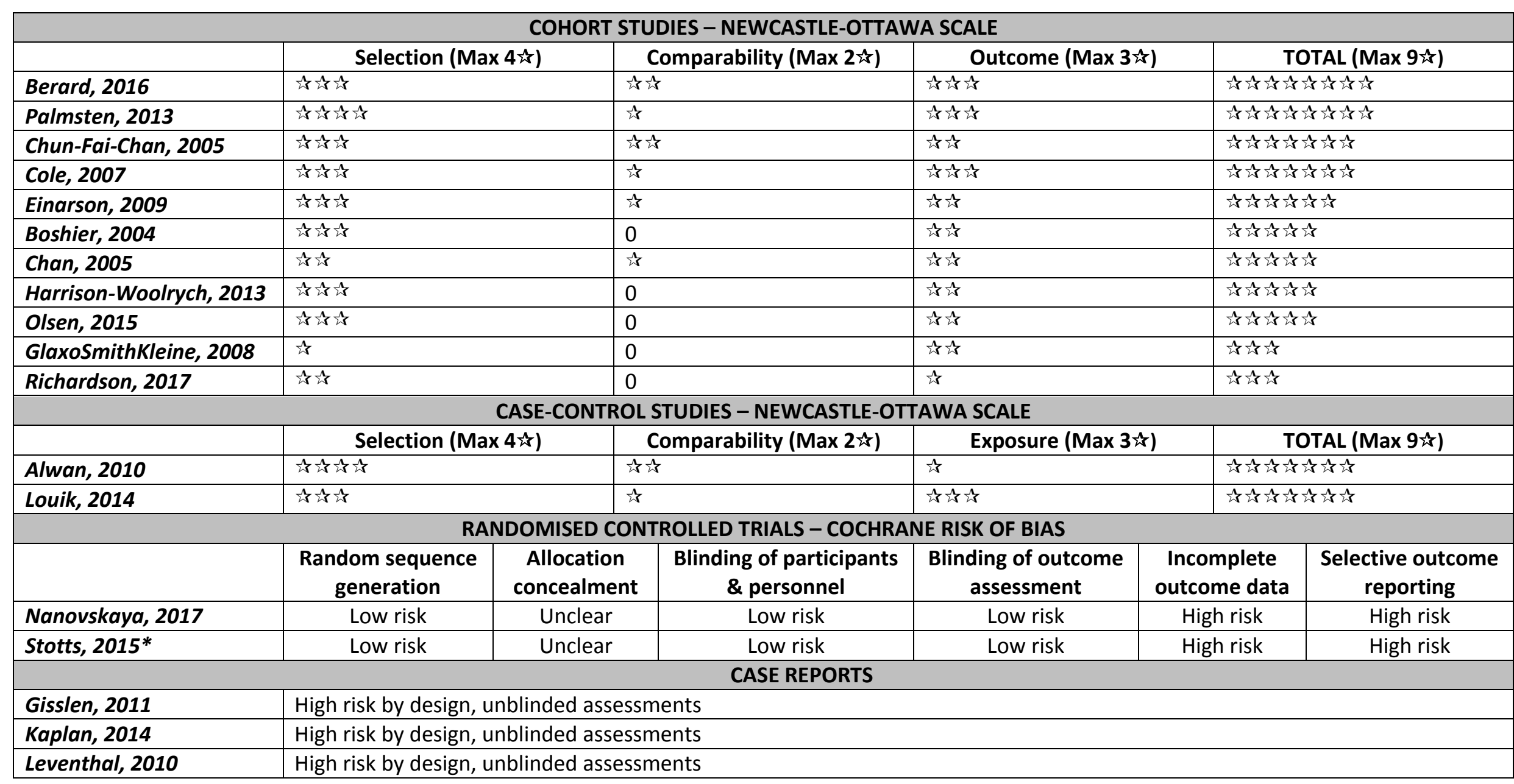

*Quality assessment for Stotts 2015 as assessed in the Cochrane Review “Pharmacological interventions for promoting smoking cessation during pregnancy" (2015) ${ }^{10}$ 